\title{
Article \\ Relativistic B-Spline R-Matrix Calculations for Electron Scattering from Thallium Atoms
}

\author{
Yang Wang ${ }^{1, *}$, Hai-Liang Du ${ }^{1}$, Xi-Ming Zhu ${ }^{1,2}$, Oleg Zatsarinny ${ }^{3,+}+$ and Klaus Bartschat $^{3, *}$ \\ 1 School of Physics, Harbin Institute of Technology, Harbin 150001, China; dhl6494@outlook.com (H.-L.D.); \\ simon.ximing.zhu@outlook.com (X.-M.Z.) \\ 2 School of Energy Science and Engineering, Harbin Institute of Technology, Harbin 150001, China \\ 3 Department of Physics and Astronomy, Drake University, Des Moines, IA 50311, USA \\ * Correspondence: yangwang0624@foxmail.com (Y.W.); klaus.bartschat@drake.edu (K.B.) \\ + Deceased.
}

check for updates

Citation: Wang, Y.; Du, H.-L.;

Zhu, X.-M.; Zatsarinny, O.;

Bartschat, K. Relativistic B-Spline R-Matrix Calculations for Electron Scattering from Thallium Atoms. Atoms 2021, 9, 94. https://doi.org/ $10.3390 /$ atoms 9040094

Academic Editor: Luca Argenti

Received: 15 October 2021

Accepted: 3 November 2021

Published: 5 November 2021

Publisher's Note: MDPI stays neutral with regard to jurisdictional claims in published maps and institutional affiliations.

Copyright: (c) 2021 by the authors. Licensee MDPI, Basel, Switzerland. This article is an open access article distributed under the terms and conditions of the Creative Commons Attribution (CC BY) license (https:// creativecommons.org/licenses/by/ $4.0 /)$.

\begin{abstract}
The Dirac B-spline R-matrix (DBSR) method is employed to treat low-energy electron collisions with thallium atoms. Special emphasis is placed on spin polarization phenomena that are investigated through calculations of the differential cross-section and the spin asymmetry function. Overall, good agreement between the present calculations and the available experimental measurements is found. The contributions of electron exchange to the spin asymmetry cannot be ignored at low impact energies, while the spin-orbit interaction plays an increasingly significant role as the impact energy rises.
\end{abstract}

Keywords: electron scattering; thallium; elastic; excitation; differential cross-section; spin asymmetry function; fully relativistic Dirac B-spline R-matrix

\section{Introduction}

Spin polarization phenomena in electron-atom collisions have been of great interest for many years [1]. Compared to cross-sections alone, they provide a deeper insight into the importance of spin-dependent interactions in collision processes, such as spin-orbit and electron exchange effects. The left-right spin asymmetry function $S_{A}$ is a measure of the spin dependence of the differential cross-section (DCS) in collisions of spin-polarized electrons with a given target. It is a very sensitive parameter to test the quality of a theoretical approach to electron-atom collisions $[2,3]$. This is particularly true for excitation processes, and the difficulties increase significantly with the complexity of the target. For open-shell heavy targets, significant contributions can be attributed to both electron exchange via the so-called "fine-structure effect" [4] and the spin-orbit interaction of the continuum electron in the field of the target atom (Mott scattering). The competition between them makes $S_{A}$ extremely sensitive to the details of the theoretical approach.

Thallium (Tl) is a challenging target with a heavy nucleus $(Z=81)$. It has an open-shell $6 s^{2} 6 p$ with nonvanishing orbital angular momentum as its ground-state configuration. Benchmark experimental data for e- $\mathrm{Tl}$ collisions at low energies were produced by the Münster group [5-7]. Kaussen et al. [5] investigated the spin polarization in elastic scattering of unpolarized electrons from several heavy atoms, including thallium, for selected incident-electron energies between 6 and $180 \mathrm{eV}$. Geesmann et al. [6] used a source of spin-polarized electrons, which were scattered elastically and inelastically from an ensemble of unpolarized thallium atoms, to study the spin asymmetry function for incident energies ranging from 3 to $14 \mathrm{eV}$ and scattering angles in the range of $35^{\circ}$ to $125^{\circ}$. Later, the measurements were extended to energies below $2 \mathrm{eV}$ by Dümmler et al. [7].

On the theoretical side, Bartschat and collaborators [8-12] performed semi-relativistic Breit-Pauli R-matrix (BPRM) calculations for elastic and inelastic electron scattering from thallium atoms. Their results showed that both the fine-structure effect and Mott scattering 
could be important for an open-shell heavy atom such as Tl. A fully relativistic generalized density functional (GDF) was developed by Haberland and Fritsche [13] and Fritsche et al. [14]. They concluded that the fine-structure effect only has a small influence at low impact energies. Later, Bostock et al. [15] employed the relativistic convergent-closecoupling (RCCC) method to calculate the spin asymmetries for elastic and inelastic cases. They suggested that the spin asymmetries can be mediated by relativistic effects (spin-orbit interactions) in addition to exchange effects.

In the present paper, we aim to study the relative influence of Mott scattering and the fine-structure effect for both elastic and inelastic e-Tl scattering. We employ the fully relativistic Dirac B-spline R-matrix (DBSR) method developed by Zatsarinny to perform calculations in a 33-state close-coupling model. In addition to the spin asymmetry function $S_{A}$, the angle-differential cross-sections (DSCs) are also presented below.

This manuscript is organized as follows. In Section 2, we briefly summarize the main aspects of our computational models. This is followed with results for energy levels and oscillator strengths in Section 3.1, the angle-DCS in Section 3.2, and the spin asymmetry function $S_{A}$ in Section 3.3. We finish with some conclusions in Section 4.

\section{Computational Method}

The present calculations employed the fully relativistic DBSR method. It is based on the close-coupling expansion for the total $(e+\mathrm{Tl})$ wave function for the collision system. A write-up of the semi-relativistic version of the method and the associated computer program was published by Zatsarinny [16] and the extension to the fully relativistic framework was described in detail by Zatsarinny and Bartschat [17]. Examples of its application can be found in the review [18].

In the present DBSR calculations, we chose 33 discrete states of $\mathrm{Tl}$ in the close-coupling expansion, with dominant configurations $6 s^{2} n l(l=3$ for $n=5, l=1-3$ for $n=6, l=0-3$ for $n=7$ and $8, l=0-2$ for $n=9$ and 10) and $6 s 6 p^{2}$, respectively. The number of physical states that we can generate in this method depends upon the size of the R-matrix box. The R-matrix radius was set to $60 a_{0}\left(a_{0}=0.529 \times 10^{-10} \mathrm{~m}\right.$, Bohr radius $)$ and the target Hamiltonian was diagonalized in this box. This choice allowed us to obtain a sufficiently good description for the low-lying bound states of Tl up to $6 s^{2} 8 f$ (see Table 1 in Section 3.1). All Dirac-Fock calculations for the core and the correlated orbitals were performed with the relativistic atomic structure packages GRASP2K [19] and DBSR_HF [20]. Different sets of correlated $n l$ orbitals were optimized separately for the lowest state of each symmetry with total target electronic angular momentum and parity $(n l) J^{\pi}$.

We employed 123 B-splines of orders 8 and 9 for the large and small components of the spinors, respectively. The different B-spline orders for the two components ensure the absence of spurious solutions [21]. The relatively large number of splines is due to the fact that we employed a finite-size nuclear model with a Fermi potential. Partialwave contributions up to $J=50$ were numerically calculated. This number of partial waves is sufficient for the present cases of interest. No top-up procedure to estimate contributions from higher partial waves was therefore needed. The present calculations included up to 146 coupled scattering channels, and interaction matrices with dimensions up to approximately 36,000 needed to be diagonalized for each partial wave. In order to perform these calculations, we employed the parallelized DBSR code and also used parallelized linear-algebra libraries such as SCALAPACK. The calculation in the external region was performed with the FARM program [22]. Finally, the scattering amplitudes, the DCS, and the spin asymmetry function $S_{A}$ were determined by using the formulas given, for example, in Refs. [3,23].

Specifically, $S_{A}$ is expressed as

$$
\frac{\sigma_{\text {left }}(E, \theta)-\sigma_{\text {right }}(E, \theta)}{\sigma_{\text {left }}(E, \theta)+\sigma_{\text {right }}(E, \theta)} \equiv S_{A}(E, \theta) P_{\perp},
$$


where $P_{\perp}$ is the spin polarization component perpendicular to the scattering plane, $\sigma$ is the DCS, $\theta$ is the scattering angle, $E$ is the projectile energy, and left/right are determined with respect to the incident beam axis in the scattering plane defined by the initial and final projectile momenta, respectively. We employed the MJK program [24] to generate these observables from the transition matrix elements after ensuring the appropriate phase conventions of the spherical harmonics in the different programs. An approximate test for the importance of the fine-structure effect in spin polarization phenomena is the average value of the spin asymmetry function $S_{A}$ [12], which can be obtained as

$$
<S_{A}>=\frac{\sum_{J_{1} J_{0}}\left(2 J_{0}+1\right) \sigma^{J_{0} \rightarrow J_{1}}(E, \theta) S_{A}^{J_{0} \rightarrow J_{1}}(E, \theta)}{\sum_{J_{1}, J_{0}}\left(2 J_{0}+1\right) \sigma^{J_{0} \rightarrow J_{1}}(E, \theta)} .
$$

Here, $J_{0}$ and $J_{1}$ denote the total angular momenta of the target in its initial and final states, respectively, during the transitions. In the non-relativistic limit, electron exchange still has contributions to $S_{A}$, while Mott scattering does not. Therefore, the pure fine-structure effect is considered as a non-relativistic approximation, in which the spin-orbit interaction is neglected. Then, the energy levels of a pair of fine-structure states are equal. As a result, the average values of spin asymmetry function $S_{A}$ will vanish. A detailed discussion of the fine-structure effect can be found in the review by Hanne [4].

\section{Results and Discussion}

\subsection{Energy Levels and Oscillator Strengths}

Table 1 shows the energy levels of $\mathrm{Tl}$ obtained in the present calculation. The excitation energies from the ground state are compared with those given in the NIST [25] tables. The overall agreement between the present results and the NIST tables is satisfactory, with the deviations in energy splitting being generally less than $0.1 \mathrm{eV}$ for most states. The energies of the $6 s 6 p^{2}{ }^{4} P_{1 / 2}, 6 s^{2} 10 d{ }^{2} D_{3 / 2,5 / 2}$, and $6 s^{2} 8 f{ }^{2} F^{\circ}{ }_{1 / 2,3 / 2}$ states have somewhat larger discrepancies above $0.2 \mathrm{eV}$. However, the deviations do not exceed $5 \%$.

Another assessment of the quality of target description in the present model can be made by comparing the results for the oscillator strengths of various transitions with available experimental data. Such a comparison is reported in Table 2 for selected excitations of $\mathrm{Tl}$ from the fine-structure levels $6 s^{2} 6 p^{2} P^{\circ}{ }_{1 / 2}$ and $6 s^{2} 6 p^{2} P_{3 / 2}^{\circ}$. Specifically, we show our theoretical values obtained in the velocity $(\mathrm{V})$ and length $(\mathrm{L})$ forms of the electric dipole operator. The experimental values are from the data recommended by NIST [25], and references [26] (obtained with the optical-double-resonance technique, ODRT) and [27] (employing beam-foil technique, BFT). The present results are in good to moderate agreement with the experimental data. In some cases, deviations of up to $40 \%$ are seen, with the results obtained in the length gauge generally being larger than those obtained in the velocity gauge. The most likely reason for the deviations between the two sets as well as the recommended data is the computational necessity to limit the number of configurations in the target description in a way that makes the subsequent collision calculation possible.

\subsection{Differentical Cross-Sections}

The present DBSR predictions of the DCS are shown in Figure 1 for elastic electron collisions from $\mathrm{Tl}$ atoms in their ground state $6 s^{2} 6 p^{2} P^{\circ} 1 / 2$ for incident electron energies between 1 and $24 \mathrm{eV}$. No experimental data are available for comparison. The magnitudes of the DCS for all incident energies are close at scattering angles smaller than 5 degrees. A systematic move of the DCS minimum is found going from around 110 degrees at $1 \mathrm{eV}$ to approximately 90 degrees at $11 \mathrm{eV}$. The DCS curves become broader and flatten out in their bottom region as the energy increases, and more minima appear when the energy is higher than $15 \mathrm{eV}$. Three significant minima appear at 20 and $24 \mathrm{eV}$. Their angular positions are around 40, 90, and 130 degrees, respectively. The curves for energies higher than $11 \mathrm{eV}$ intersect near 145 degrees. 
Table 1. Energy levels of $\mathrm{Tl}$ (in eV) relative to the ground state obtained in this work, compared with those in the NIST [25] tables ( $J$ denotes the total angular momentum quantum number).

\begin{tabular}{|c|c|c|c|c|c|c|}
\hline No. & State & Term & $J$ & NIST [25] & This Work & Difference \\
\hline 1 & $6 s^{2} 6 p$ & ${ }^{2} P^{\circ}$ & $1 / 2$ & 0.00000 & 0.00000 & 0.00000 \\
\hline 2 & $6 s^{2} 6 p$ & ${ }^{2} P^{\circ}$ & $3 / 2$ & 0.96617 & 0.93535 & -0.03082 \\
\hline 3 & $6 s^{2} 7 s$ & ${ }^{2} S$ & $1 / 2$ & 3.28279 & 3.35298 & 0.07019 \\
\hline 4 & $6 s^{2} 7 p$ & ${ }^{2} P^{\circ}$ & $1 / 2$ & 4.23529 & 4.26149 & 0.02620 \\
\hline 5 & $6 s^{2} 7 p$ & ${ }^{2} P^{\circ}$ & $3 / 2$ & 4.35942 & 4.30594 & -0.05348 \\
\hline 6 & $6 s^{2} 6 d$ & ${ }^{2} D$ & $3 / 2$ & 4.47805 & 4.47796 & -0.00009 \\
\hline 7 & $6 s^{2} 6 d$ & ${ }^{2} D$ & $5 / 2$ & 4.48822 & 4.48293 & -0.00529 \\
\hline 8 & $6 s^{2} 8 s$ & ${ }^{2} S$ & $1 / 2$ & 4.80388 & 4.81059 & 0.00671 \\
\hline 9 & $6 s^{2} 8 p$ & ${ }^{2} P^{\circ}$ & $1 / 2$ & 5.12899 & 5.12293 & -0.00606 \\
\hline 10 & $6 s^{2} 8 p$ & ${ }^{2} P^{\circ}$ & $3 / 2$ & 5.1752 & 5.15013 & -0.02507 \\
\hline 11 & $6 s^{2} 7 d$ & ${ }^{2} D$ & $3 / 2$ & 5.20875 & 5.18402 & -0.02473 \\
\hline 12 & $6 s^{2} 7 d$ & ${ }^{2} D$ & $5 / 2$ & 5.21341 & 5.17956 & -0.03385 \\
\hline 13 & $6 s^{2} 5 f$ & ${ }^{2} F^{\circ}$ & $5 / 2$ & 5.24681 & 5.22070 & -0.02611 \\
\hline 14 & $6 s^{2} 5 f$ & ${ }^{2} F^{\circ}$ & $7 / 2$ & 5.24681 & 5.22151 & -0.02530 \\
\hline 15 & $6 s^{2} 9 s$ & ${ }^{2} S$ & $1 / 2$ & 5.35193 & 5.34096 & -0.01097 \\
\hline 16 & $6 s^{2} 9 p$ & ${ }^{2} P^{\circ}$ & $1 / 2$ & 5.50253 & 5.48915 & -0.01338 \\
\hline 17 & $6 s^{2} 9 p$ & ${ }^{2} P^{\circ}$ & $3 / 2$ & 5.52505 & 5.50476 & -0.02029 \\
\hline 18 & $6 s^{2} 8 d$ & ${ }^{2} D$ & $3 / 2$ & 5.5387 & 5.51819 & -0.02051 \\
\hline 19 & $6 s^{2} 8 d$ & ${ }^{2} D$ & $5 / 2$ & 5.54119 & 5.51857 & -0.02262 \\
\hline 20 & $6 s^{2} 6 f$ & ${ }^{2} F^{\circ}$ & $5 / 2$ & 5.55741 & 5.53341 & -0.02400 \\
\hline 21 & $6 s^{2} 6 f$ & ${ }^{2} F^{\circ}$ & $7 / 2$ & 5.55741 & 5.53366 & -0.02375 \\
\hline 22 & $6 s 6 p^{2}$ & ${ }^{4} P$ & $1 / 2$ & 5.607 & 5.32811 & -0.27889 \\
\hline 23 & $6 s^{2} 10 s$ & ${ }^{2} S$ & $1 / 2$ & 5.61609 & 5.61945 & 0.00336 \\
\hline 24 & $6 s^{2} 10 p$ & ${ }^{2} P^{\circ}$ & $1 / 2$ & 5.69575 & 5.74420 & 0.04845 \\
\hline 25 & $6 s^{2} 10 p$ & ${ }^{2} P^{\circ}$ & $3 / 2$ & 5.70868 & 5.76181 & 0.05313 \\
\hline 26 & $6 s^{2} 9 d$ & ${ }^{2} D$ & $3 / 2$ & 5.71549 & 5.75791 & 0.04242 \\
\hline 27 & $6 s^{2} 9 d$ & ${ }^{2} D$ & $5 / 2$ & 5.71695 & 5.75781 & 0.04086 \\
\hline 28 & $6 s^{2} 7 f$ & ${ }^{2} F^{\circ}$ & $5 / 2$ & 5.72625 & 5.75303 & 0.02678 \\
\hline 29 & $6 s^{2} 7 f$ & ${ }^{2} F^{\circ}$ & $7 / 2$ & 5.72625 & 5.75313 & 0.02688 \\
\hline 30 & $6 s^{2} 10 d$ & ${ }^{2} D$ & $3 / 2$ & 5.82105 & 6.05811 & 0.23706 \\
\hline 31 & $6 s^{2} 10 d$ & ${ }^{2} D$ & $5 / 2$ & 5.82205 & 6.06582 & 0.24377 \\
\hline 32 & $6 s^{2} 8 f$ & ${ }^{2} F^{\circ}$ & $5 / 2$ & 5.82783 & 6.03291 & 0.20508 \\
\hline 33 & $6 s^{2} 8 f$ & ${ }^{2} F^{\circ}$ & $7 / 2$ & 5.82783 & 6.03290 & 0.20507 \\
\hline
\end{tabular}

Table 2. Selected oscillator strengths for excitation of $\mathrm{Tl}$ from $6 s^{2} 6 p$ to upper levels, as obtained in the velocity (V) and length (L) forms of the electric dipole operator. (The subscript number denotes the total electronic angular momentum quantum number).

\begin{tabular}{ccccccc}
\hline Lower & Upper & & & & \multicolumn{2}{c}{ This Work } \\
\cline { 6 - 7 } Level & Level & NIST [25] & ODRT [26] & BFT [27] & V & L \\
\hline $6 s^{2} 6 p_{1 / 2}$ & $6 s^{2} 6 d_{3 / 2}$ & 0.29 & $0.29 \pm 0.022$ & 0.34 & 0.2271 & 0.3307 \\
& $6 s^{2} 7 s_{1 / 2}$ & 0.134 & $0.133 \pm 0.007$ & 0.13 & 0.1276 & 0.1399 \\
& $6 s^{2} 7 d_{3 / 2}$ & 0.075 & $0.074 \pm 0.009$ & 0.09 & 0.0555 & 0.0814 \\
& $6 s^{2} 8 s_{1 / 2}$ & 0.018 & $0.0176 \pm 0.0016$ & & 0.0169 & 0.0199 \\
& $6 s^{2} 8 d_{3 / 2}$ & & $0.028 \pm 0.004$ & & 0.0237 & 0.0331 \\
& $6 s^{2} 9 s_{1 / 2}$ & & $0.0062 \pm 0.0008$ & & 0.0050 & 0.0050 \\
$6 s^{2} 6 p_{3 / 2}$ & $6 s^{2} 6 d_{3 / 2}$ & 0.0411 & $0.04 \pm 0.004$ & & 0.0349 & 0.0499 \\
& $6 s^{2} 6 d_{5 / 2}$ & 0.346 & $0.346 \pm 0.035$ & 0.37 & 0.3100 & 0.4273 \\
& $6 s^{2} 7 s_{1 / 2}$ & 0.151 & $0.151 \pm 0.007$ & 0.14 & 0.1295 & 0.1870 \\
& $6 s^{2} 7 d_{3 / 2}$ & & $0.0091 \pm 0.0009$ & & 0.0079 & 0.0109 \\
& $6 s^{2} 7 d_{5 / 2}$ & 0.08 & $0.081 \pm 0.009$ & 0.08 & 0.0619 & 0.0858 \\
& $6 s^{2} 8 s_{1 / 2}$ & 0.0135 & $0.0136 \pm 0.0014$ & & 0.0118 & 0.0172 \\
& $6 s^{2} 8 d_{3 / 2}$ & & $0.004 \pm 0.0004$ & & 0.0031 & 0.0043 \\
& $6 s^{2} 8 d_{5 / 2}$ & & $0.028 \pm 0.003$ & 0.03 & 0.0233 & 0.0316 \\
& $6 s^{2} 9 s_{1 / 2}$ & & $0.0048 \pm 0.0005$ & & 0.0027 & 0.0043 \\
& $6 s^{2} 9 d_{3 / 2}$ & & $0.002 \pm 0.0002$ & & 0.0025 & 0.0035 \\
& $6 s^{2} 10 s_{1 / 2}$ & & $0.003 \pm 0.0003$ & & 0.0023 & 0.0035 \\
\hline
\end{tabular}




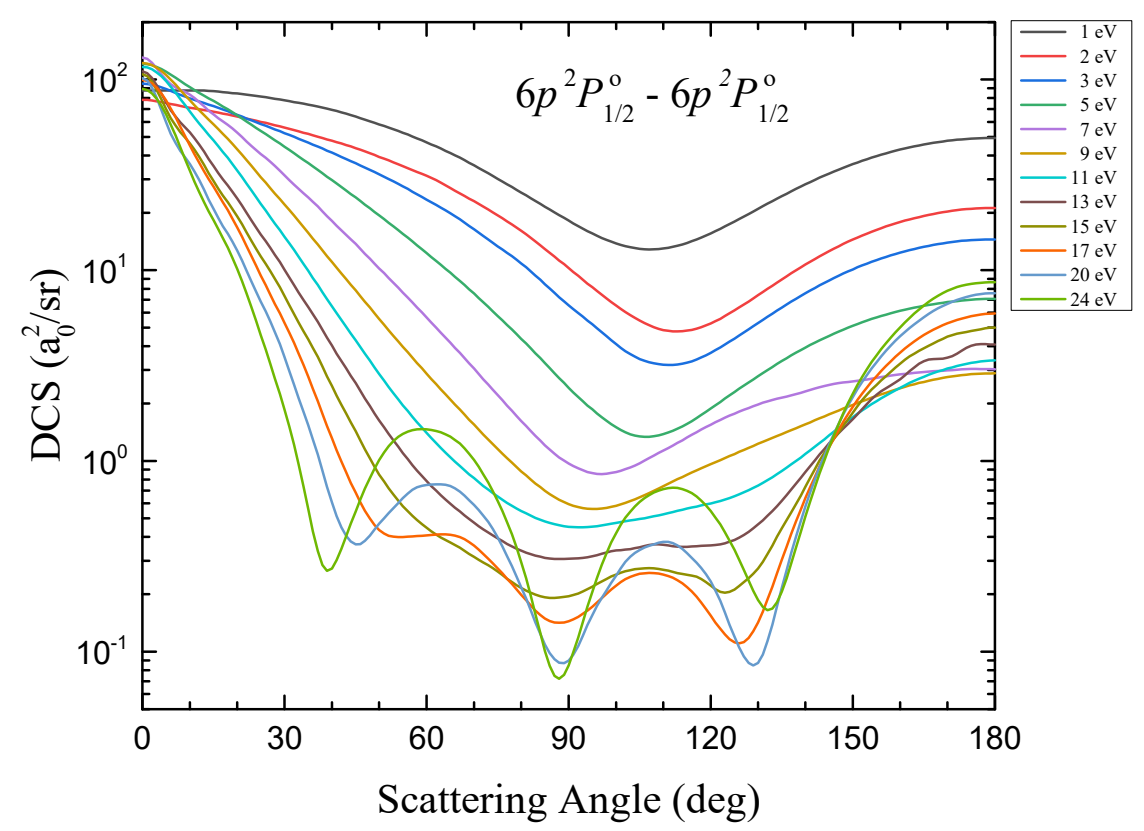

Figure 1. DCS for elastic electron collisions from $\mathrm{Tl}$ atoms in their $6 s^{2} 6 p^{2} P^{\circ} 1 / 2$ ground state for a variety of projectile energies.

Figure 2 depicts the corresponding results for electron impact excitation of the $6 s^{2} 6 p$ ${ }^{2} P^{\circ}{ }_{3 / 2}$ state from the ground state. It is not surprising that the DCSs for the inelastic transitions are generally much smaller than for the elastic case. Most of the curves exhibit peaks at scattering angles of around 7 degrees, where the values of the DCSs at different impact energies are very similar. Unlike for the elastic case, the minima in these curves show a systematic movement towards the direction of larger angles, and turn into a single deep valley near 90 degrees for incident energies higher than $15 \mathrm{eV}$.

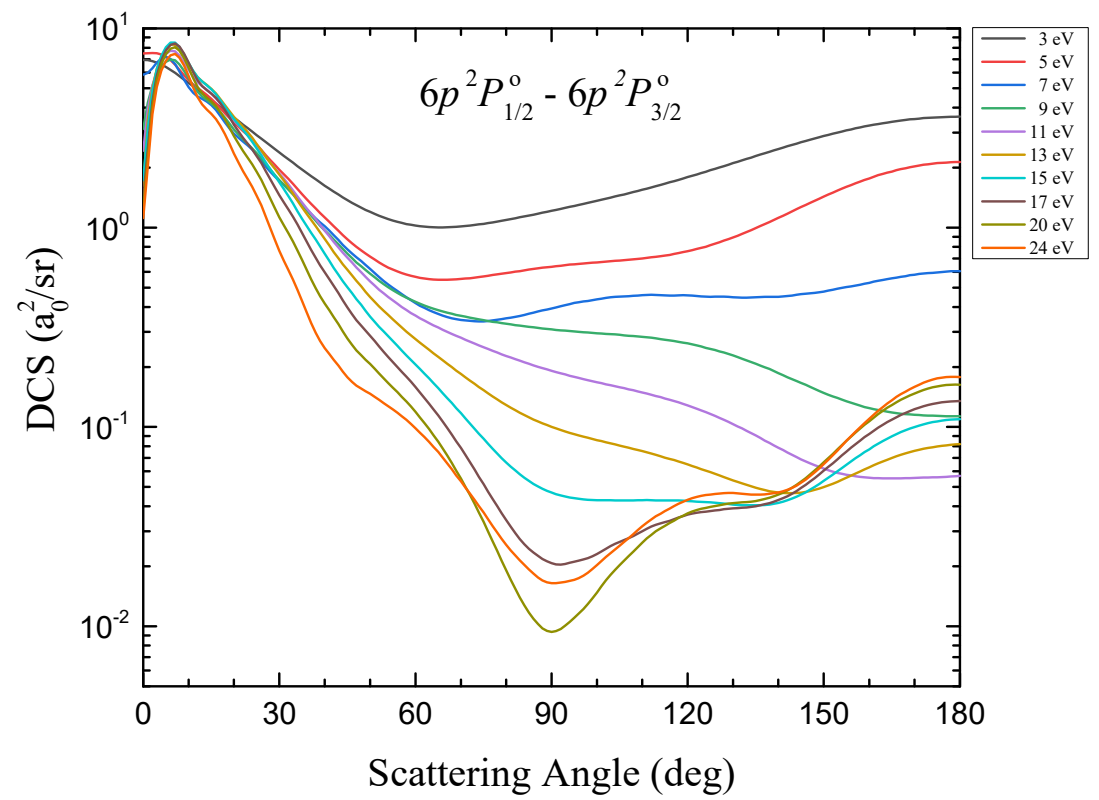

Figure 2. DCS for electron impact excitation of the $6 s^{2} 6 p^{2} P_{1 / 2}^{\circ} \rightarrow 6 s^{2} 6 p^{2} P_{3 / 2}^{\circ}$ transition in $\mathrm{Tl}$ for a variety of projectile energies. 
The case for electron impact excitation of the $6 s^{2} 7 s^{2} S_{1 / 2}$ state from the ground state exhibits a different dependence in the curves of DCSs compared to the former two cases, which is displayed in Figure 3. The DCSs decrease rapidly from 0 to 15 degrees, especially for the higher impact energies. Most curves intersect at scattering angles of around 15 degrees. There is still a systematic movement of the DCS minima towards smaller angles. The DCSs for excitation from the ground state to $6 s^{2} 7 s^{2} S_{1 / 2}$ have generally a smaller magnitude than those for excitation from the ground state to $6 s^{2} 6 p^{2} P_{3 / 2}^{\circ}$. However, this is not the case near the forward direction at scattering angles smaller than 15 degrees. The deep valleys also emerge in Figure 3 near 90 and 130 degrees for incident energies higher than $17 \mathrm{eV}$.

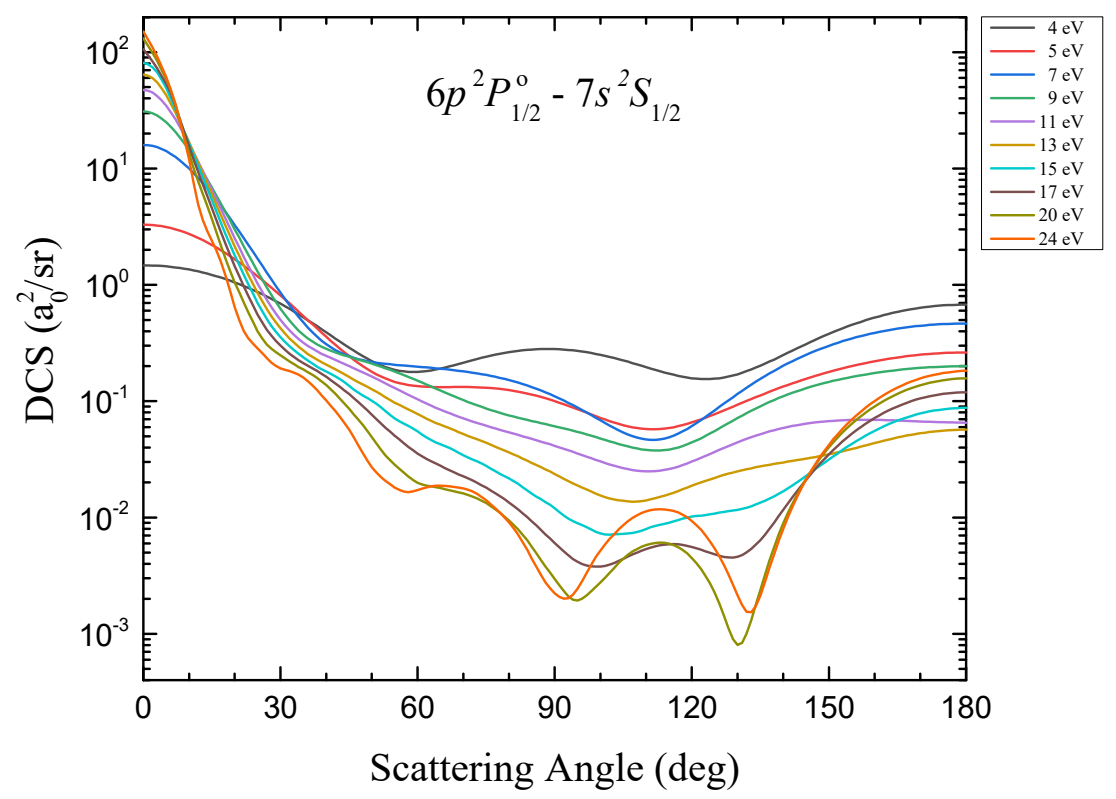

Figure 3. DCS for electron impact excitation of the $6 s^{2} 6 p^{2} P^{\circ}{ }_{1 / 2} \rightarrow 6 s^{2} 7 s^{2} S_{1 / 2}$ transition in $\mathrm{Tl}$ for a variety of projectile energies.

\subsection{Spin Asymmetries}

Figure 4 exhibits the spin asymmetry function $S_{A}$ for elastic electron collisions from Tl atoms in the ground state $6 s^{2} 6 p^{2} P_{1 / 2}^{\circ}$ for incident electron energies between 1 and $24 \mathrm{eV}$. The present DBSR predictions are compared with the experimental data of the Münster group [5-7], along with the theoretical results of BPRM method by Bartschat [11] and Goerss et al. [12], GDF calculations by Haberland and Fritsche [13], as well as the RCCC predictions by Bostock et al. [15]. The present DBSR prediction results slightly overestimate the values of $S_{A}$ at scattering angles near 90 degrees for the cases of low impact energies displayed in Figure 4. However, it is clear that the present results are still in overall good agreement with the experimental data, especially for the cases at higher energies such as at $17 \mathrm{eV}$ and $24 \mathrm{eV}$. One should note that there are three narrow peaks in the $S_{A}$ curve for $24 \mathrm{eV}$ at scattering angles near 40,90, and 135 degrees. These positions coincide with the three significant minima in the corresponding DCS curve for $24 \mathrm{eV}$, displayed in Figure 1. This is a typical characteristic of Mott scattering, where high values of $\left|S_{A}\right|$ are correlated with minima in the DCS. This suggests that the dominant contribution to the polarization mechanism is the spin-orbit interaction in this case. In contrast, such characteristic correlations between the DCS and $S_{A}$ are not so evident for the low incident energies below $6 \mathrm{eV}$ shown in Figure 4. This indicates that electron exchange processes may also play an important role for the spin polarization in elastic e-Tl collisions at low energies. 


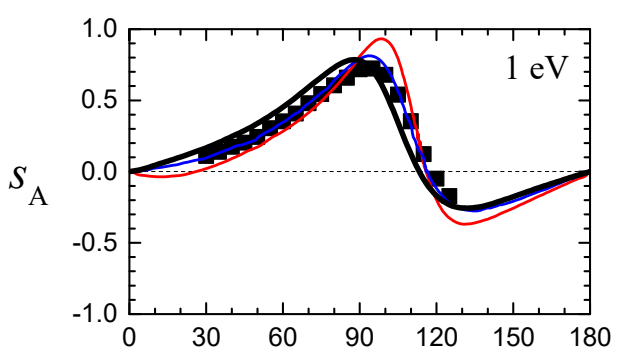

$6 p^{2} P_{1 / 2}^{o}-6 p{ }^{2} P_{1 / 2}^{o}$

$\longrightarrow$ DBSR

- Expt. Dümmler et al.

- Expt. Geesmann et al.

$\triangle$ Expt. Kaussen et al.
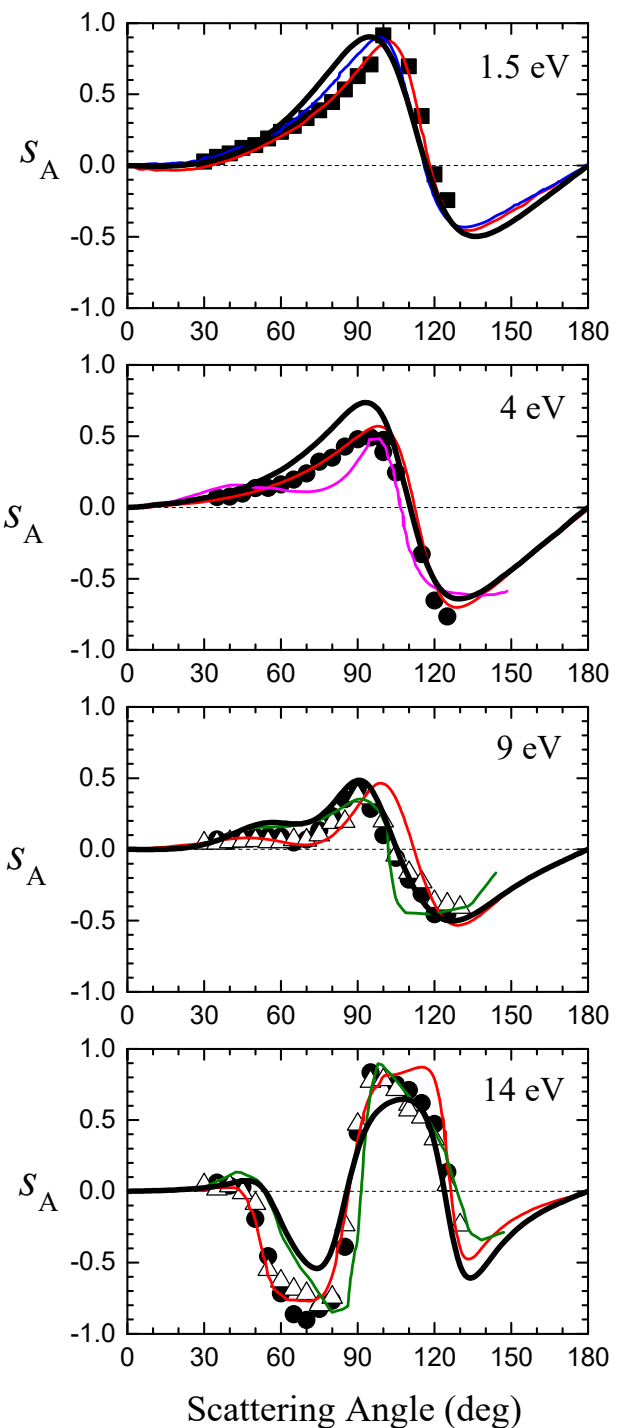
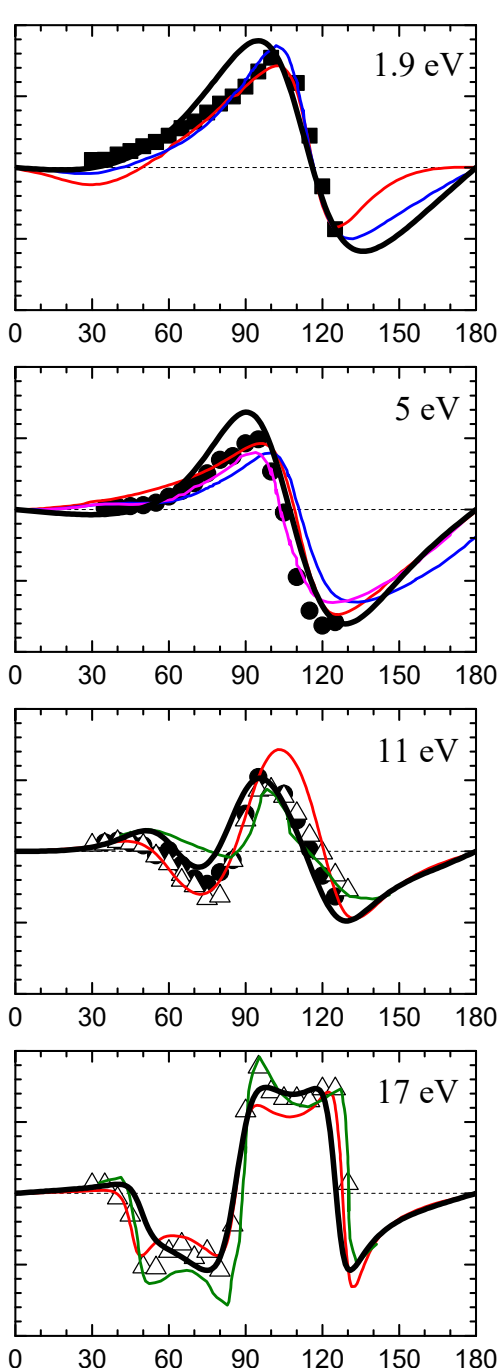

Scattering Angle (deg)
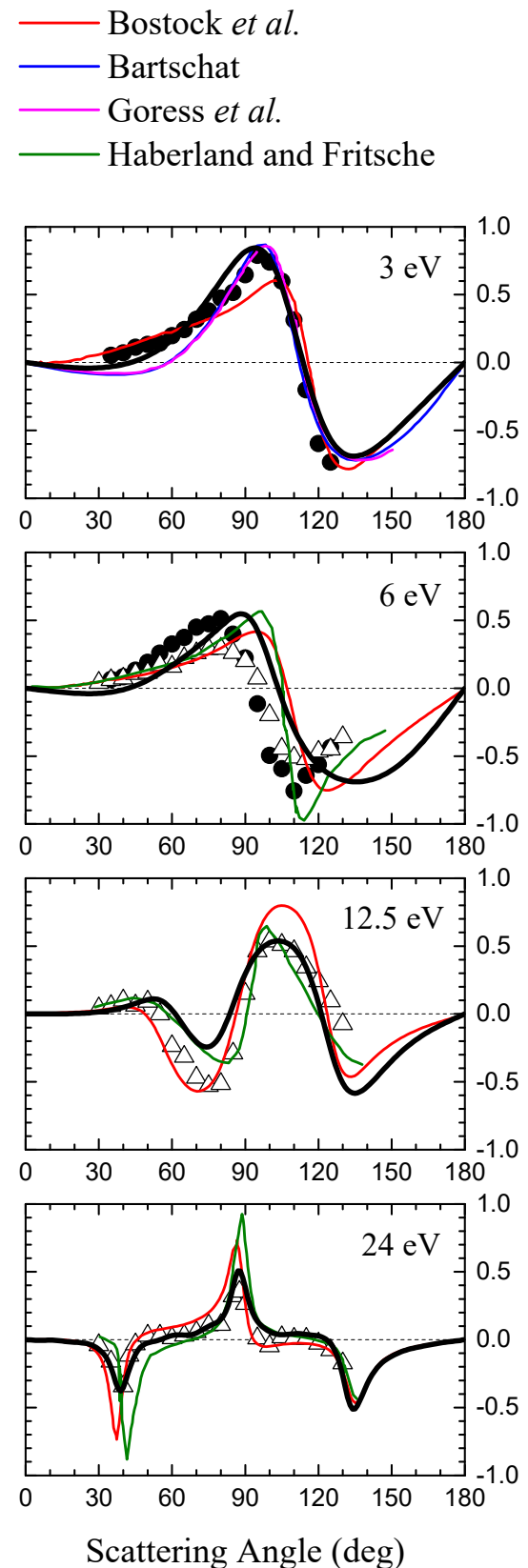

Figure 4. Spin asymmetry function $S_{A}$ for elastic electron collisions from Tl atoms in the ground state $6 s^{2} 6 p^{2} P^{\circ}{ }_{1 / 2}$ for incident electron energies between 1 and $24 \mathrm{eV}$. The DBSR results are compared with the experimental data of Kaussen et al. [5], Geesmann et al. [6], and Dümmler et al. [7], along with the theoretical results of Bartschat [11], Goerss et al. [12], Haberland and Fritsche [13], and Bostock et al. [15].

Figures 5 and 6 exhibit the DBSR results for the spin asymmetry function $S_{A}$ for electron impact excitation of $\mathrm{Tl}$ atoms from their ground state $6 s^{2} 6 p^{2} P^{\circ}{ }_{1 / 2}$ to the $6 s^{2} 6 p$ ${ }^{2} P_{3 / 2}^{\circ}$ and $6 s^{2} 7 s^{2} S_{1 / 2}$ states, respectively. We also show the experimental measurements of Geesmann et al. [6], along with RCCC calculations by Bostock et al. [15] and BPRM calculations by Goerss et al. [12]. The present calculations represent a clear improvement over the semi-relativistic BPRM results. This indicates that relativistic effects should be fully considered when studying electron scattering from heavy atoms such as thallium, especially 
for excitation. The agreement between the present DBSR results and the experimental measurements is generally good.

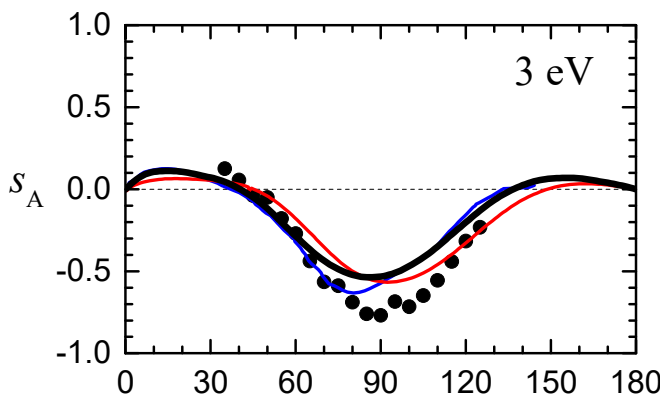

$$
6 p{ }^{2} P_{1 / 2}^{o}-6 p{ }^{2} P_{3 / 2}^{o}
$$

DBSR

Bostock et al.

Goress et al.

- Expt. Geesmann et al.
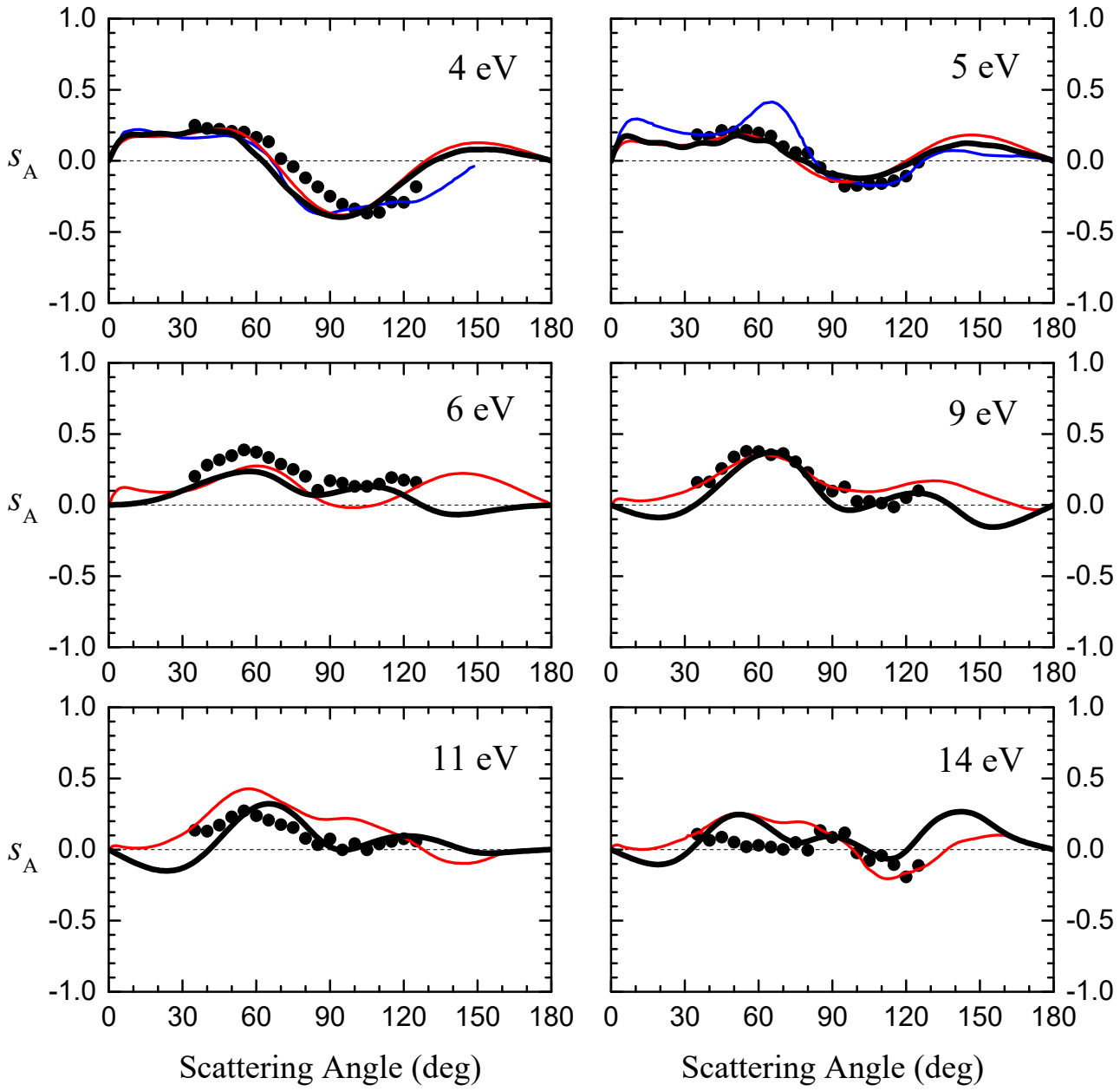

Figure 5. Spin asymmetry function $S_{A}$ for electron impact excitation of the $6 s^{2} 6 p^{2} P^{\circ}{ }_{1 / 2} \rightarrow 6 s^{2} 6 p$ ${ }^{2} P_{3 / 2}^{\circ}$ transition in $\mathrm{Tl}$ for incident energies between 3 and $14 \mathrm{eV}$. The DBSR results are compared with the experimental data of Geesmann et al. [6], along with the theoretical results of Goerss et al. [12] and Bostock et al. [15]. 

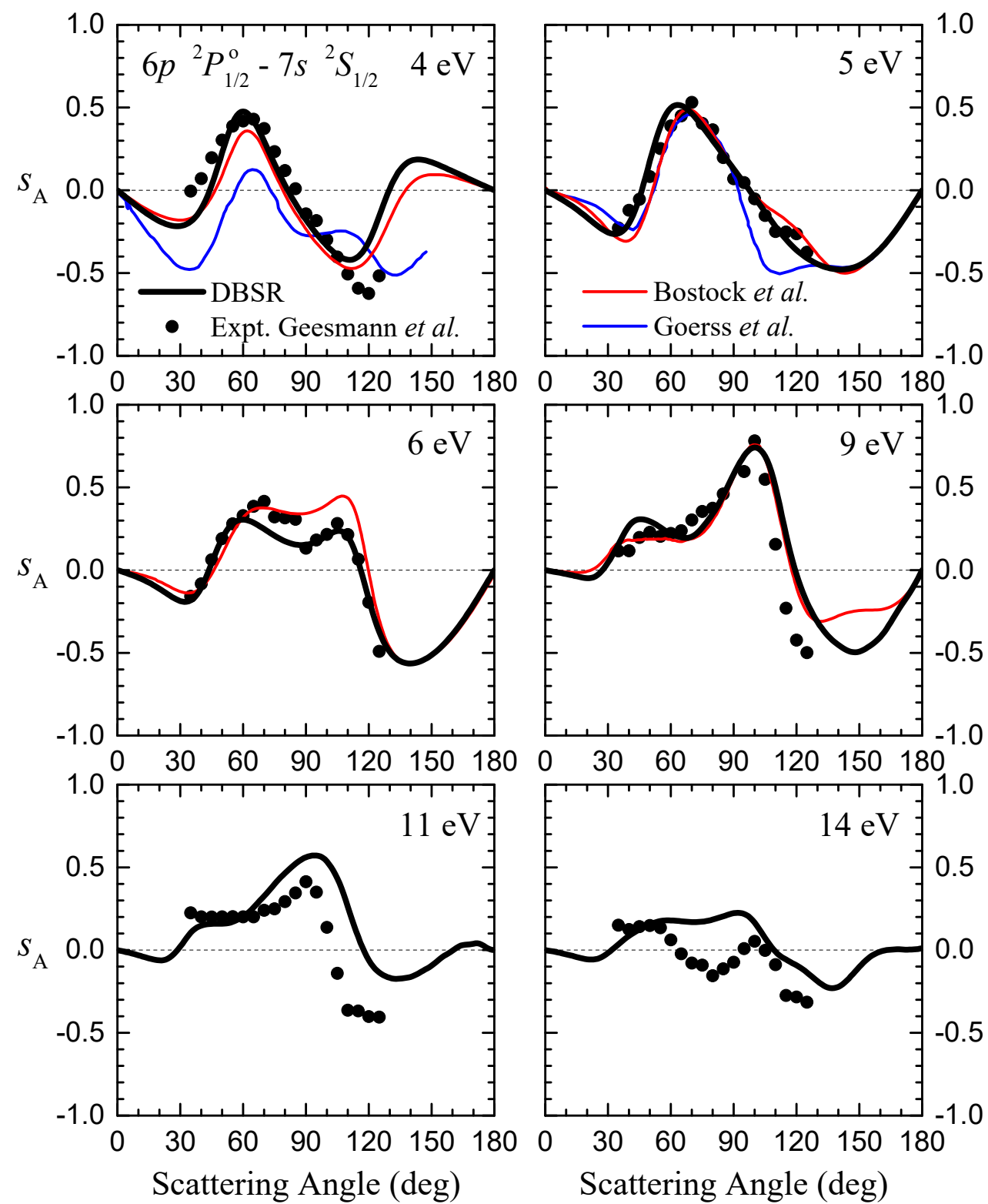

Figure 6. Spin asymmetry function $S_{A}$ for electron impact excitation of the $6 s^{2} 6 p^{2} P^{\circ} 1 / 2 \rightarrow 6 s^{2} 7 s$ ${ }^{2} S_{1 / 2}$ transition in $\mathrm{Tl}$ for incident energies between 4 and $14 \mathrm{eV}$. The DBSR results are compared with the experimental data of Geesmann et al. [6], along with the theoretical results of Goerss et al. [12] and Bostock et al. [15].

The average values of $S_{A}$ were calculated according to Equation (2) for the transitions between the fine-structure levels of the ground-state configuration $6 s^{2} 6 p^{2} P^{\circ}$ and the excited-state configurations $6 s^{2} 7 s^{2} S, 6 s^{2} 7 p^{2} P^{\circ}$, and $6 s^{2} 6 d^{2} D$, respectively. Figure 7 depicts the present DBSR predictions for incident electron energies of 5, 7.5, 10, 12.5, and $15 \mathrm{eV}$, along with the theoretical results of the BPRM method by Goerss et al. [12]. We also show $S_{A}$ for individual fine-structure transitions from the $6 s^{2} 6 p^{2} P^{\circ}{ }_{1 / 2}$ and $6 s^{2} 6 p^{2} P^{\circ}{ }_{3 / 2}$ states, respectively, to the $6 s^{2} 7 s^{2} S_{1 / 2}$ state at $5 \mathrm{eV}$. The average $S_{A}$ values for transitions from $6 s^{2} 6 p$ ${ }^{2} P^{\circ}$ to $6 s^{2} 7 s{ }^{2} S$ are significantly smaller than the values for the individual fine-structure transitions. The situation is similar for the other two sets of transitions, in agreement with the findings of Goerss et al. [12]. This indicates that the fine-structure effect cannot be ignored for these cases. Especially for the $6 s^{2} 6 p^{2} P^{\circ}$ to $6 s^{2} 6 d^{2} D$ transitions, the angular dependence of the average $S_{A}$ is almost flat, while significant contributions to the spin asymmetry by the fine-structure effect are possible in the fine-structure-resolved transitions. 
On the other hand, the average $S_{A}$ values for each set of transitions show a stronger angular dependence as the impact energy increases. This suggests that the spin-orbit interaction plays a more important role at higher energies.

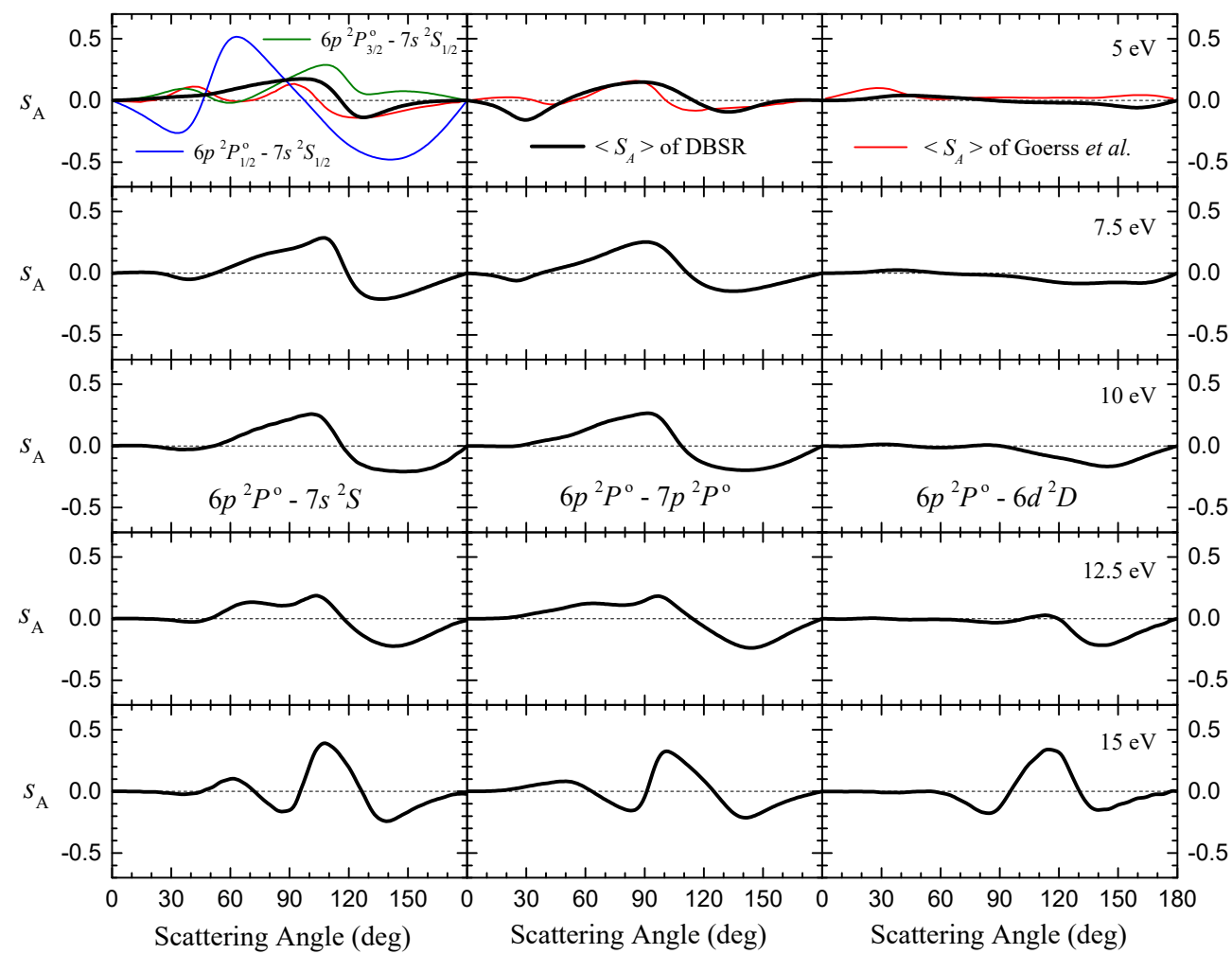

Figure 7. Average spin asymmetry function $S_{A}$ for electron impact excitation of the $6 s^{2} 6 p^{2} P^{\circ} \rightarrow 6 s^{2} 7 s$ ${ }^{2} S, 6 s^{2} 6 p^{2} P^{\circ} \rightarrow 6 s^{2} 7 p^{2} P^{\circ}$ and $6 s^{2} 6 p^{2} P^{\circ} \rightarrow 6 s^{2} 6 d^{2} D$ transitions in $\mathrm{Tl}$ for incident energies between 5 and $15 \mathrm{eV}$. The DBSR results are compared with the theoretical results of Goerss et al. [12]. As one example for comparison, fine-structure-resolved predictions are shown in the top left panel.

\section{Conclusions}

In this paper, we have presented calculations of the differential cross-section and the spin asymmetry functions for elastic scattering and electron impact excitation to the $6 s^{2} 6 p$ ${ }^{2} P_{3 / 2}^{\circ}$ and $6 s^{2} 7 s^{2} S_{1 / 2}$ states in thallium atoms. Our ab initio calculations are based on the fully relativistic Dirac B-spline R-matrix (DBSR) approach that Oleg Zatsarinny developed for years. The excellent agreement between theoretical predictions and experimental measurements indicates, once again, the reliability of the DBSR method to treat the process of electron-atom collisions. We also conclude that, at low energies, even for the openshell heavy target thallium, the spin polarization in the electron scattering process can still be attributed to a significant extent to electron exchange effects, in addition to the spin-orbit interaction.

Author Contributions: Conceptualization, Y.W., X.-M.Z. and K.B.; methodology, O.Z. and K.B.; software, O.Z.; calculations Y.W. and H.-L.D.; data curation, H.-L.D.; writing-original draft preparation, Y.W., H.-L.D. and K.B.; writing-review and editing, Y.W. and K.B.; project administration, X.-M.Z.; funding acquisition, X.-M.Z. All authors have read and agreed to the published version of the manuscript.

Funding: This research was funded by the National Natural Science Foundation of China under grant no. 11775063, and the Defense Industrial Technology Development Program under grant no. JCKY2018203B029. The work of O.Z. and K.B. was supported by the United States National Science Foundation under grants no. PHY-1903844, OAC-1834740, and PHY-2110023. 
Data Availability Statement: Tabulated results of the cross-sections are available from the authors upon request.

Acknowledgments: We thank the HEPC Studio at the Physics School of the Harbin Institute of Technology for access to high-performance computing resources through INSPUR-HPC@hepc.hit.edu.cn.

Conflicts of Interest: The authors declare no conflict of interest. The funders had no role in the design of the study; in the collection, analyses, or interpretation of data; in the writing of the manuscript, or in the decision to publish the results.

\section{References}

1. Kessler, J. Polarized Electrons, 2nd ed.; Springer: New York, NY, USA, 1985.

2. Andersen, N.; Bartschat, K.; Broad, J.T.; Hertel, I.V. Collisional alignment and orientation of atomic outer shells: III. Spin-resolved excitation. Phys. Rep. 1997, 279, 251-396. [CrossRef]

3. Andersen, N.; Bartschat, K. Polarization, Alignment, and Orientation in Atomic Collisions; Springer: New York, NY, USA, 2001.

4. Hanne, G.F. Spin effects in inelastic electron-atom collisions. Phys. Rep. 1983, 95, 95-165. [CrossRef]

5. Kaussen, F.; Geesmann, H.; Hanne, G.F.; Kessler, J. Study of spin polarisation in elastic scattering of electrons from $\mathrm{Hg}$, $\mathrm{Tl}, \mathrm{Pb}$ and Bi atoms. J. Phys. B At. Mol. Phys. 1987, 20, 151. [CrossRef]

6. Geesmann, H.; Bartsch, M.; Hanne, G.F.; Kessler, J. Left-right asymmetry for scattering of polarized electrons from thallium and lead atoms. J. Phys. B At. Mol. Opt. Phys. 1991, 24, 2817. [CrossRef]

7. Dummler, M.; Bartsch, M.; Geesmann, H.; Hanne, G.F.; Kessler, J. Low-energy data of the Sherman function of $\mathrm{Hg}$, $\mathrm{Tl}$ and $\mathrm{Pb}$. J. Phys. B At. Mol. Opt. Phys. 1992, 25, 4281. [CrossRef]

8. Bartschat, K.; Scott, N.S. Resonances in the low-energy scattering of electrons by atomic thallium. J. Phys. B At. Mol. Phys. 1984, 17, 3787. [CrossRef]

9. Bartschat, K.; Blum, K.; Burke, P.G.; Hanne, G.F.; Scott, N.S. The fine-structure effect in the low-energy scattering of electrons on $\mathrm{Hg}$ and Tl atoms. J. Phys. B At. Mol. Phys. 1984, 17, 3797. [CrossRef]

10. Bartschat, K.; Goerss, H.-J.; Nordbeck, R.-P. Generalized STU-parameters for elastic electron scattering from thallium and lead atoms. Z. Phys. D-At. Mol. Clust. 1990, 17, 25-31. [CrossRef]

11. Bartschat, K. Close-coupling calculations for low-energy elastic from thallium atoms. Z. Phys. D-At. Mol. Clust. 1994, 30, 85-90. [CrossRef]

12. Goerss, H.-J.; Nordbeck, R.-P.; Bartschat, K. Generalized STU parameters for elastic and inelastic low-energy scattering of electrons from thallium atoms. J. Phys. B At. Mol. Opt. Phys. 1991, 24, 2833. [CrossRef]

13. Haberland, R.; Fritsche, L. On the elastic scattering of low-energy electrons by Hg, Tl, Pb and Bi atoms. J Phys. B At. Mol. Phys. 1987, 20, 121. [CrossRef]

14. Fritsche, L.; Kroner, C.; Reinert, T. A consistent relativistic theory of electron scattering by atoms. J. Phys. B At. Mol. Opt. Phys. 1992, 25, 4287. [CrossRef]

15. Bostock, C.J.; Fursa, D.V.; Bray, I. Relativistic convergent close-coupling calculation of spin asymmetries for electron-thallium scattering. Phys. Rev. A 2012, 86, 062701. [CrossRef]

16. Zatsarinny, O. BSR: B-spline atomic R-matrix codes. Comput. Phys. Commun. 2006, 174, 273-356. [CrossRef]

17. Zatsarinny, O.; Bartschat, K. Relativistic B-spline R-matrix method for electron collisions with atoms and ions: Application to low-energy electron scattering from Cs. Phys. Rev. A 2008, 77, 062701. [CrossRef]

18. Zatsarinny, O.; Bartschat, K. The B-spline R-matrix method for atomic processes: Application to atomic structure, electron collisions, and photoionization. J. Phys. B At. Mol. Opt. Phys. 2013, 46, 112001. [CrossRef]

19. Jönsson, P.; He, X.; Fischer, C.F.; Grant, I.P. The grasp2K relativistic atomic structure package. Comput. Phys. Commun. 2007, 177, 597-622. [CrossRef]

20. Zatsarinny, O.; Fischer, C.F. DBSR_HF: A B-spline Dirac-Hartree-Fock program. Comput. Phys. Commun. 2016, 202, 287-303. [CrossRef]

21. Fischer, C.F.; Zatsarinny, O. A B-spline Galerkin method for the Dirac equation. Comput. Phys. Commun. 2009, 180, 879-886. [CrossRef]

22. Burke, V.M.; Noble, C.J. Farm-A flexible asymptotic R-matrix package. Comput. Phys. Commun. 1995, 85, 471-500. [CrossRef]

23. Bartschat, K. Excitation and ionization of atoms by interaction with electrons, positrons, protons and photons. Phys. Rep. 1989, 180, 1-81. [CrossRef]

24. Grum-Grzhimailo, A.N. MJK: A program to calculate observable quantities in electron-atom collisions. Comput. Phys. Commun. 2003, 152, 101-119. [CrossRef]

25. Kramida, A.; Ralchenko, Y.; Reader, J.; NIST ASD Team. NIST Atomic Spectra Database (Ver. 5.8). 2020. Available online: https:/ / physics.nist.gov/asd (accessed on 29 September 2021).

26. Gallagher, A.; Lurio, A. Thallium Oscillator Strengths and $6 \mathrm{~d}^{2} \mathrm{D}_{3 / 2}$ State hfs. Phys. Rev. A 1964, 136, 87. [CrossRef]

27. Andersen, T.; Sørensen, G. Systematic study of atomic lifetimes in gallium, indium, and thallium measured by the beam-foil technique. Phys. Rev. A 1972, 5, 2447. [CrossRef] 